\title{
Specific oligomerization of the 5-HT1A receptor in the plasma membrane
}

\author{
Andrew Woehler • Jakub Wlodarczyk • \\ Evgeni G. Ponimaskin
}

Received: 26 June 2008 /Revised: 1 September 2008 / Accepted: 2 September 2008 / Published online: 14 October 2008

(C) Springer Science + Business Media, LLC 2008

\begin{abstract}
In the present study we analyze the oligomerization of the 5-HT1A receptor within living cells at the subcellular level. Using a 2-excitation Förster Resonance Energy Transfer (FRET) method combined with spectral microscopy we are able to estimate the efficiency of energy transfer based on donor quenching as well as acceptor sensitization between CFP-and YFP-tagged 5-HT1A receptors at the plasma membrane. Through the analysis of the level of apparent FRET efficiency over the various relative amounts of donor and acceptor, as well as over a range of total surface expressions of the receptor, we verify the specific interaction of these receptors. Furthermore we study the role of acylation in this interaction through measurements of a palmitoylation-deficient $5-\mathrm{HT}_{1 \mathrm{~A}}$ receptor mutant. Palmitoylation increases the tendency of a
\end{abstract}

\author{
A. Woehler $\cdot$ E. G. Ponimaskin $(\bowtie)$ \\ Department of Neuro and Sensory Physiology, \\ University of Göttingen, \\ Gottingen, Germany \\ e-mail: eponima@gwdg.de \\ J. Wlodarczyk \\ Max-Planck Institute for Biophysical Chemistry, \\ Gottingen, Germany \\ J. Wlodarczyk \\ Department of Molecular and Cellular Neurobiology, \\ Nencki Institute, \\ Warsaw, Poland \\ E. G. Ponimaskin \\ DFG-Research Center for the Molecular Physiology of the Brain \\ (CMPB), \\ Gottingen, Germany \\ E. G. Ponimaskin \\ Cellular Neurophysiology, Hannover Medical School, \\ Hannover, Germany
}

receptor to localize in lipid rich microdomains of the plasma membrane. This increases the effective surface density of the receptor and provides for a higher level of stochastic interaction.

Keywords Palmitoylation - Serotonin $\cdot 5$-HT1A receptor . Lipid rafts $\cdot$ FRET $\cdot$ Oligomerization
Abbreviations
GPCR G-protein coupled receptor
$E f_{D} \quad$ Donor dependent apparent FRET efficiency
$E f_{A} \quad$ Acceptor dependent apparent FRET efficiency
$x_{D} \quad$ Donor mole fraction
$T_{D} \quad$ Relative total Concentration

\section{Introduction}

G-protein coupled receptors (GPCRs) are a superfamily of proteins that govern the reactions to a wide range of signals via activation of the heterotrimeric G-proteins. Structurally, GPCRs possess seven transmembrane domains linked by alternating intracellular (i1-i3) and extracellular (e1-e4) loops. The extracellular receptor surface, including the Nterminus, is known to be critically involved in ligand binding. The intracellular receptor surface, including Cterminal domain and intracellular loops, is known to be important for G-protein recognition and activation [1]. Members of GPCR family are usually glycosylated and possess at least one glycosylation site on extracellular Nterminus. Functionally, N-linked glcosylation of GPCRs have been shown to be evolved in the regulation of the receptor's expression and functions (for review see [2]). Another common post-translational modification of GPCRs 
is the covalent attachment of palmitic acid to cysteine residues located within their cytoplasmic C-terminus [3]. Palmitoylation of several GPCRs has been shown to play a central role in the regulation of the receptor's functions [4]. Previously, our group has demonstrated that the 5-HT1A receptor is stably palmitoylated at its C-terminal cysteine residues Cys417 and Cys420. Furthermore, we have shown that palmitoylation of 5-HT1A receptor is necessary for Giprotein coupling and effector signaling [5].

In recent years there has been a shift in the understanding of distribution of GPCRs in the plasma membrane. Originally, it was believed that GPCRs existed and functioned as monomeric units. However, it is now widely accepted that in many cases oligomerization of these receptors occurs $[6,7]$. Interestingly, receptor oligomerization seems to be independent of glycosylation, as has been shown for human hisamine $\mathrm{H}(4)$ and alpha- $1 \mathrm{~b}$ adrenoreceptors $[8,9]$. In some cases this specific interaction occurs between the same receptor type (homo-oligomerization) and in others between different receptors (heterooligomerization). For several GPCRs, oligomerizaiton has been found to occur early in the receptors lifetime, shortly after translation. On the other hand, oligomerization has also been shown to occur at the plasma membrane both in ligand-dependent and independent manner [10, 11]. Functionally, homo-and hetero-oligomerization have been found to play an important role in receptor trafficking, ligand specificity and coupling to specific signaling pathways [12-17]. Thus, oligomerization may provide an additional level of control for signal transduction and for the fine tuning of cellular processes.

Another area of interest in the study of GPCRs is their lateral distribution within the plasma membrane. Rather than being uniformly distributed throughout the plasma membrane, many studies suggest that GPCRs may localize in cholesterol-rich membrane microdomains or lipid rafts [18-20]. Many of these reports, often based on biochemical assay as well as fluorescence techniques, propose that compartmentalization is necessary for the efficient interaction between signaling partners with low surface concentration. Generally, it has been suggested that lipid rafts may represent "hot spots" for signaling, working as a platform for co-localization of GPCRs with corresponding Gproteins. In addition, it has been proposed that compartmentalization in cholesterol rich microdomains occurs due to hydrophobic mismatch of transmembrane domains as well as by post-translational acylation of both the receptor and G-proteins [21-23].

In addition to the role of palmitoylation in the coupling of the receptor with G-protein [5], we have recently demonstrated the importance of this modification for the localization of the 5-HT1A receptor in the lipid rafts [24], implying that palmitoylation plays a central role in the 5-
HT1A function. We have also recently demonstrated specific homo-oligomerization of the 5-HT1A receptors [25]. In this previous investigation we applied both classical biochemical assays (e.g. co-immunoprecipitation and crosslinking) as well as biophysical approaches, including acceptor-photobleaching FRET, time correlated singlephoton counting (TCSPC) FRET, as well as, a novel FRET approach, lux-FRET, allowing for measurements of the donor-acceptor ratio and apparent FRET efficiencies from populations of living cells [26]. In the current study we applied this method to spectral images acquired using confocal microscopy, which allows for the investigation of localization and interaction of 5-HT1A receptors in living cells with high spatial resolution. Moreover, by defining a confocal slice which encompasses only the plasma membrane immediately adjacent to the coverslip, we can measure FRET signal resulting from oligomerization of 5HT1A receptors specifically at the membrane without any contamination from fluorescence from receptors localized in other intercellular compartments.

\section{Materials and methods}

\section{Adherent cell culture and transfection}

Mouse N1E-115 neuroblastoma cells from the American Type Culture collection (ATCC) were grown in Dulbecco's modified Eagle's medium (DMEM) supplemented with $10 \%$ fetal calf serum (FCS) and $1 \%$ penicillin/streptomycin at $37^{\circ} \mathrm{C}$ under $5 \% \mathrm{CO} 2$. For transient transfection, cells were seeded at low-density in on $35-\mathrm{mm}$ cover-slips and transfected with vectors encoding CFP-and YFP-tagged wild-type or acylation-deficient 5-HT1A receptors using Lipofectemine2000 Reagent (Invitrogen) according to the manufacture's instruction. Four hours after transfection, cells were serum starved overnight before analysis.

Live cell confocal microscopy and spectral imaging

Coverslips with N1E-115 cells co-expressing 5-HT1A-CFP and 5-HT1A-YFP fusion proteins were placed in a custom made image acquisition chamber in $2 \mathrm{ml}$ of D-PBS at room temperature. Images were acquired with an LSM 510-Meta confocal microscope (Carl Zeiss Jena) using a 40×/1.3 NA oil-immersion objective with a pixel count of $256 \times 256$, a pixel dwell time of $1.92 \mu \mathrm{s}$, and $4 \times$ frame averaging, resulting in a total image acquisition time of approximately $1 \mathrm{~s}$. The 458 and $488 \mathrm{~nm}$ lines of a $40 \mathrm{~mW}$ argon laser were used for the two excitation FRET method as described in [26]. Fluorescence emission was collected in eight channels of the Zeiss Meta detector at $21.4 \mathrm{~nm}$ steps. All images were digitized/collected with 12 bit resolution. 


\section{FRET analysis}

Prior to FRET analysis, images collected at two excitation wavelengths were spatially aligned with respect to each other, in order to eliminate artifacts resulting from misalignment. Image registration usually required less than a 2 pixel shift in the $x-y$ plane. Next the spectral data from each pixel of the two images were analyzed according to the method described by Wlodarczyk et al. [26]. Briefly, for each pixel of the spectral images of the FRET samples, linear unmixing was performed using CFP, YFP and background reference spectra to separate the respective contributions of these species to total fluorescence. Using the apparent concentration of the donor and acceptor fluorophores along with calibration information about the relative level of excitation of our donor and acceptor at the two different wavelengths, we are able to calculate donor apparent FRET efficiency, $E f_{D}$, acceptor apparent FRET efficiency, $E f_{A}$, the FRET corrected acceptor to donor ratio and FRET corrected total concentration with respect to either reference measurement. All images and procedures were analyzed using Matlab 7.2 (The MathWorks, Natick, MA, USA).

\section{Model of FRET in oligomers}

A model characterizing apparent FRET efficiency, $E f_{D}$, as a function of donor mole fraction, $x_{D}$, for oligomeric structures has been developed previously [27],

$E f_{D}=E\left(1-x_{d}^{n-1}\right)$.

Fitting this model to experimental data allows for the estimation of the true transfer efficiency, $E$, and also provides information about the number of units, $n$, interacting in the oligomeric complex. Recently, this model has been slightly augmented for use with $E f_{A}$, [28],

$E f_{A}=E \frac{x_{d}}{1-x_{d}}\left(1-x_{d}^{n-1}\right)$.

\section{Results and discussion}

Measurements of FRET signal from the plasma membrane of living cells

We have recently verified the oligomerization state of the 5HT1A receptor by using a co-immunoprecipitation and cross-linker assays performed in neuroblastoma N1E-115 cells [25]. These biochemical methods represent the classical approaches used for the detection of GPCR oligomerization. However, these methods often result in artificial aggregation of receptors and do not allow for the analysis of GPCR oligomerization in living cells [29]. To overcome these limitations, we have implemented a novel lux-FRET approach allowing for oligomerization analysis in living cells [26]. Despite the advantages of this method, it has only been applied to the populations of cells and therefore has not allowed for the differentiation between receptors localized at the plasma membrane and those residing in the endoplasmic reticulum, Golgi network or in transport vesicles.

In the present study we are primarily interested in the oligomerization of 5-HT1A receptors at the plasma membrane due to its possible influence on ligand binding and selectivity, G-protein coupling and activation, and receptor internalization. For these reasons it is important to verify receptor oligomerization specifically at the plasma membrane and exclude artifacts from interaction in other intercellular compartments. Therefore, we adapted the luxFRET approach to the application at confocal microscopy, allowing for the analysis of receptor oligomerization with sub-cellular resolution. Figure 1A shows the 3-D reconstruction of a series of confocal slices through an N1E-115 cell co-expressing 5-HT1A-CFP and 5-HT1A-YFP receptor constructs at a 1:1 ratio. The center panel shows the confocal slice where fluorescence was detected specifically at the plasma membrane immediately adjacent to the coverslip on which the cell is growing. In addition to the clear membrane localization of the fluorescently-tagged receptors, some intercellular fluorescence is also detectable (particularly apparent in the y-z section). In the following FRET measurements similar confocal slices were used for analysis. To avoid artefacts resulting from overexpression, we adjusted the total expression level for the CFP-and YFPtagged receptor to $1.000-1.200 \mathrm{fmol} / \mathrm{mg}$ proteins in all following FRET experiments, which allows for quantitative analysis of results obtained in different experiments [25]. Moreover, similar amounts of endogenous 5-HT1A receptors has been obtained in hippocampus under physiological conditions $[30,31]$.The lux-FRET method implemented in this investigation requires the acquisition of fluorescent emission spectra resulting from two separate excitation wavelengths. To apply this method to microscopy, two images were acquired, one with excitation using the $458 \mathrm{~nm}$ laser line, the other with excitation at $488 \mathrm{~nm}$. Fluorescence emission was collected simultaneously over eight channels for each acquisition, allowing for reconstruction of emission spectra on a per pixel basis. Figure 1B shows the measured emission intensities of a single pixel over the eight channels used in the image acquisition for a cell coexpressing 5-HT1A-CFP and 5-HT1A-YFP. This figure also shows the weighted contributions of CFP, YFP and background signal which combine to provide a fit to the acquired fluorescent spectra of the FRET sample. Using the per pixel weights of CFP and YFP from the fitting of two 
A

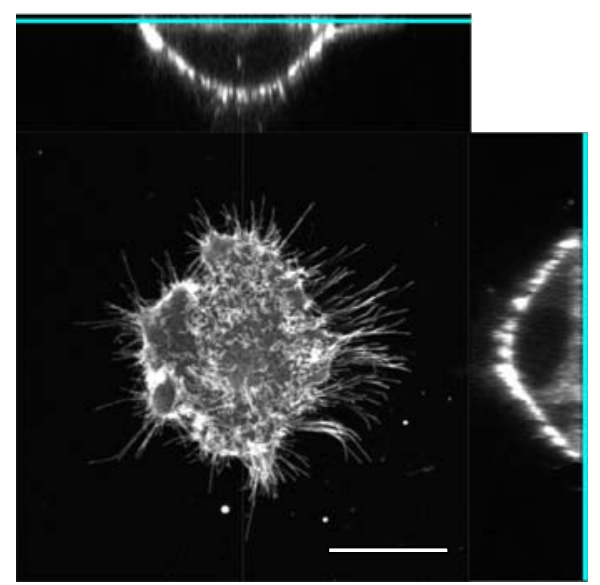

B

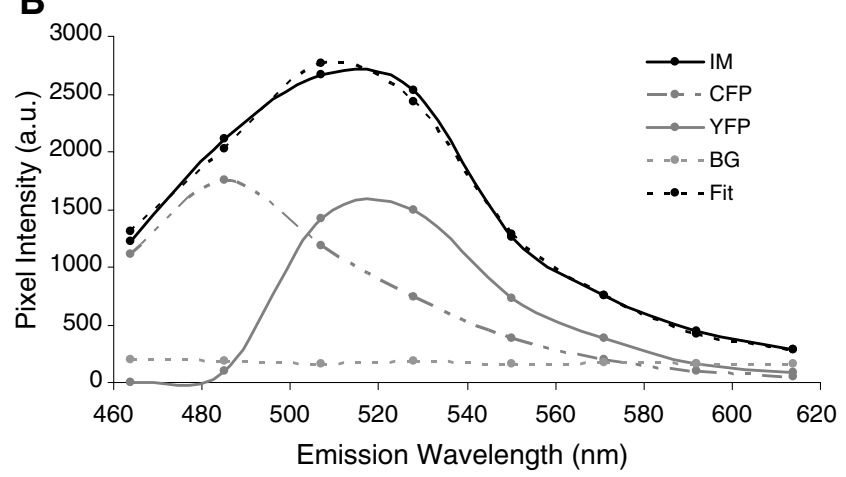

Fig 1 Overview of the method used to measure FRET selectively at the plasma membrane. (A) A series of z-stacks were acquired for a cell co-expressing YFP-tagged 5-HT1A receptor. Three-dimensional reconstruction and orthogonal plane analysis shows the receptor distribution in the $x, y$ and $z$ plane. The images show localization of the receptors in the plasma membrane as well as minor florescence from intercellular structures (most prominent in the $y-z$ plane) that can lead to incorrect characterization of FRET in whole cell measurements. The cyan lines shown in both the $x-z$ and $y-z$ planes represent the confocal depth of the $x-y$ image which is representative of those acquired for FRET analysis. Scale bar represents $20 \mu \mathrm{m}$. (B) Simultaneous acquisition of fluorescence emission in eight channels allows for the reconstruction of per pixel fluorescence emission spectra of a FRET sample co-expressing 5-HT1A-CFP and 5-HT1AYFP receptors, designated as ' $I M$ '. Linear unmixing allows us to determine the weighted contributions from CFP, YFP and the background signal that combine to fit the measured spectra, designated as 'CFP', 'YFP', 'BG', and 'Fit', respectively. The weights of the donor and acceptor spectra that provide the best fit, along with calibration parameters, are used in the analysis of FRET

emission spectra, we can apply the lux-FRET methods to define donor-and acceptor-based apparent FRET efficiencies $\left(E f_{D}\right.$ and $\left.E f_{A}\right)$, a FRET-corrected donor molar fraction $\left(x_{D}\right)$, as well as a FRET-corrected total receptor concentration $\left(T_{D}\right)$ for each pixel of the image. This allows us to not only quantify apparent FRET as a function of donor/ acceptor ratio, but also to investigate the effect of local concentration differences on oligomerization specificity at the single-cell level. It is also notable, that although the
GFP-variants used in this study do not have the monomeric mutation, this does not result in artificial FRET signal. We have previously investigated FRET resulting from the assumed spontaneous aggregation of the cytosolic nonmonomeric CFP and YFP coexpressed in neuroblastoma N1E-115 cells [26]. In this study we reported that the level of FRET in control measurements showed a strong dependence on concentration, with the maximum $E f_{D}$ value of 0.05 only occurring at a much higher concentration than was used in the current investigation.

Oligomerization of the 5-HT1A receptor in the plasma membrane is independent of its palmitoylation state

The apparent FRET efficiencies, $E f_{D}$ and $E f_{A}$, measured by the lux-FRET technique are estimates for the true energy transfer efficiency that are scaled by donor or acceptor fractional labeling. The fractional labeling terms, $f_{D}$ and $f_{A}$, are defined as the concentration of donor and acceptor in the FRET complexes, $[D A]$, divided by total respective fluorophore concentration, $\left[D^{t}\right]$ and $\left[A^{t}\right]$. The fractional labeling terms are inherently dependent on the acceptor to donor ratio. Based on this dependency, a model has been previously developed allowing for the estimation of the true energy transfer efficiency as well as the number of units interacting in an oligomeric complex [27, 28]. This model can correctly identify case of dimerization $(n=2)$ and, although it cannot accurately quantify the number of units reacting if above two, it still can be used to identify such cases.

Application of this model requires not only the measurement of apparent FRET efficiency, but also of an acceptor to donor ratio that is corrected for donor quenching as well as for acceptor sensitization. The lux-FRET provides these values and allows us to correctly apply this model to characterize the homo-oligomerization between wild-type and acylation-deficient 5-HT1A receptors. To acquire the data required to implement this model, cells grown on coverslips were co-transfected with different amounts of CFP-and YFP-tagged receptors. It is noteworthy that the total DNA concentration used for each transfection was held constant, while the ratios between donor (CFP-tagged receptors) and acceptor (YFP-tagged receptor) was varied between 1:7 and 7:1. In the image acquisition for FRET measurements, the confocal plane was adjusted so that only fluorescence from proteins localized in the plasma membrane immediately adjacent to the coverslip was collected. Initial images were acquired with the $458 \mathrm{~nm}$ laser line and immediately followed by acquisitions with the $488 \mathrm{~nm}$ laser line. Finally, FRET calculations were performed on a per pixel basis as described in the "Materials and methods" section. For the determination of the relationship between apparent FRET efficiency and acceptor to donor ratio, 115 
Fig 2 Oligomerization of the wild-type 5-HT1A receptor measured by lux-FRET. (A) Application of the two excitation FRET method described in "Materials and methods" and outlined in Fig. 1, allows us to create images of $E f_{D}, E f_{A}, x_{D}$, as well as FRET corrected total concentration $T_{D}$. (B) Mean values of $10 \times 10 \mu \mathrm{m}$ regions of interest from cells expressing different ratios of 5-HT1A-CFP and 5-HT1A-YFP receptors allows to characterize the apparent FRET efficiency over the donor mole fraction. The data were collected from 115 cells and binned along donor mole fraction in 0.05 increments. Data points represent the mean \pm SD of the apparent FRET efficiency values from each bin. The fits to the data are based on the models described in Eqs. 1 and 2. The fitted parameters from the $E f_{D}$ model were found to be $E=0.11$ and $n=2.96\left(R^{2}=0.69\right)$ and from the $E f_{A}$ model, $E=0.16 n=2.44$ $\left(R^{2}=0.83\right)$. (C) To address the effect of surface concentration on measured FRET a measured $E f_{A}$ is shown plotted as a function of corresponding relative total concentration $T_{D}$. The plot shows that $E f_{A}$ is fairly independent from the total concentration at the region of membrane at which it was measured. The data points shown represent mean $\pm \mathrm{SE}$ of $E f_{A}$ values separated by corresponding $T_{D}$ value into 15 bins. The relative total surface concentration, $T_{D}$, was calculated by the lux-FRET method and corrected for both donor quenching and acceptor sensitization (independent of FRET) and is given in units of donor reference concentration
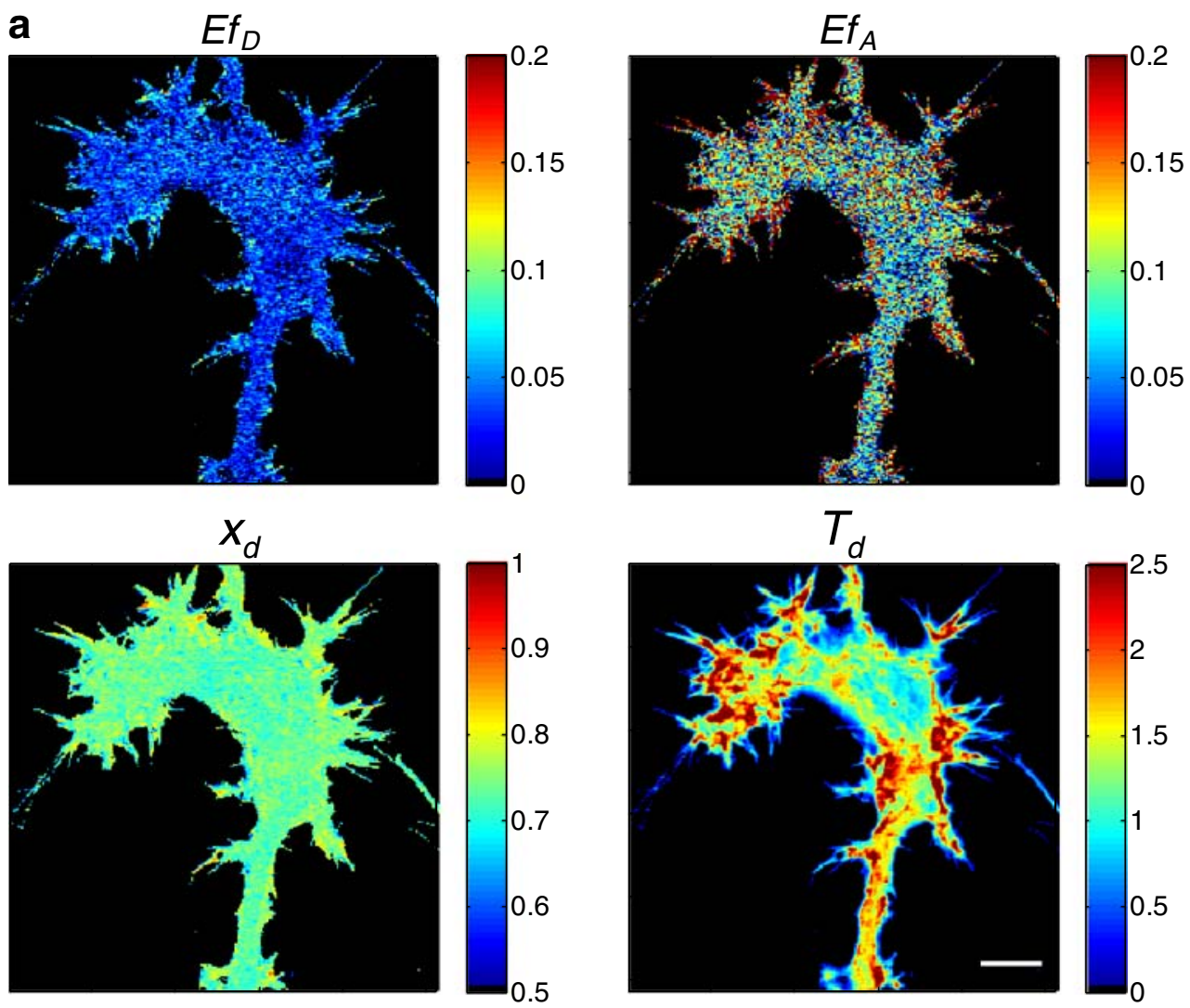

b

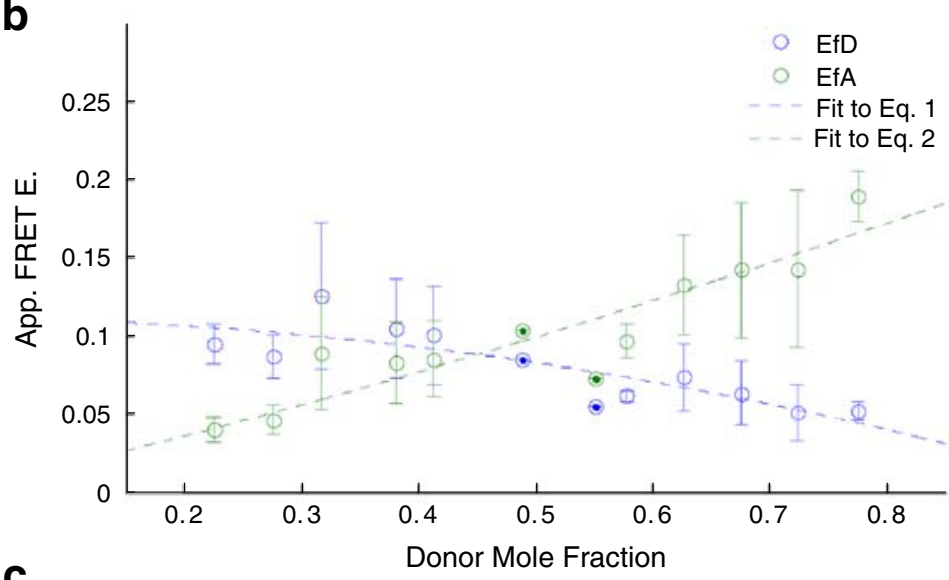

C

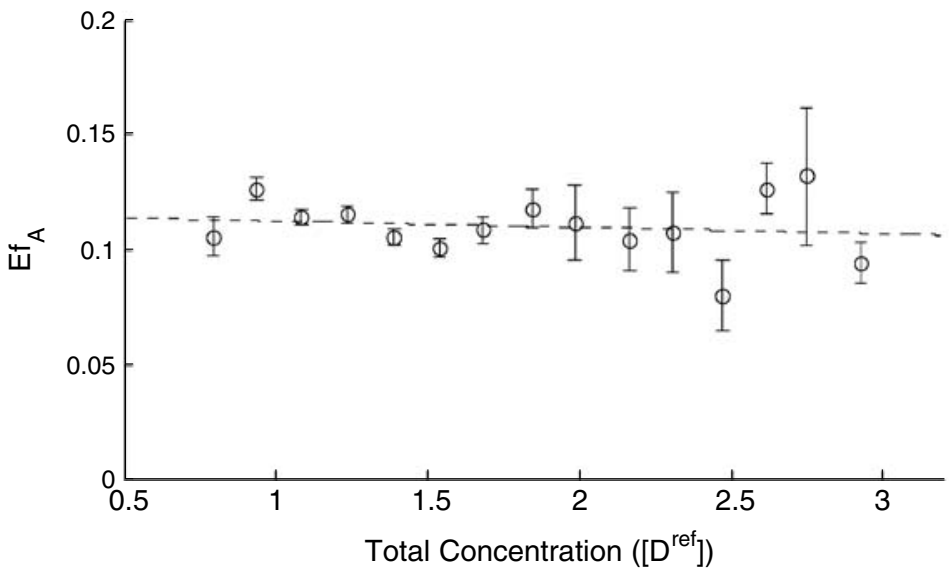


Fig 3 Oligomeriztion of the palmitoylation-deficient 5HT1A receptor measured by lux-FRET. (A) lux-FRET images of N1E-115 cells expressing palmitoylationdeficient mutant receptors show that the membrane distribution of the calculated values appear no different than for the wildtype receptors shown in Fig. 2A. (B) The characterization of FRET as a function of donor mole fraction further shows that there is little difference between the interaction of the wild type receptors and the interaction of the mutant receptors. Application of the previously mentioned models suggests that for the $E f_{D}$ data $E=0.21$ and $n=1.70\left(R^{2}=\right.$ $0.86)$ and for the $E f_{A}$ data $E=$ 0.17 and $n=2.10\left(R^{2}=0.89\right)$. (C) Similarly to the wild-type receptor, plotting $E f_{A}$ values against spatially corresponding concentration values shows that there is no dependence of measured FRET on surface concentration of the palmitoylation-deficient 5HT1A receptor
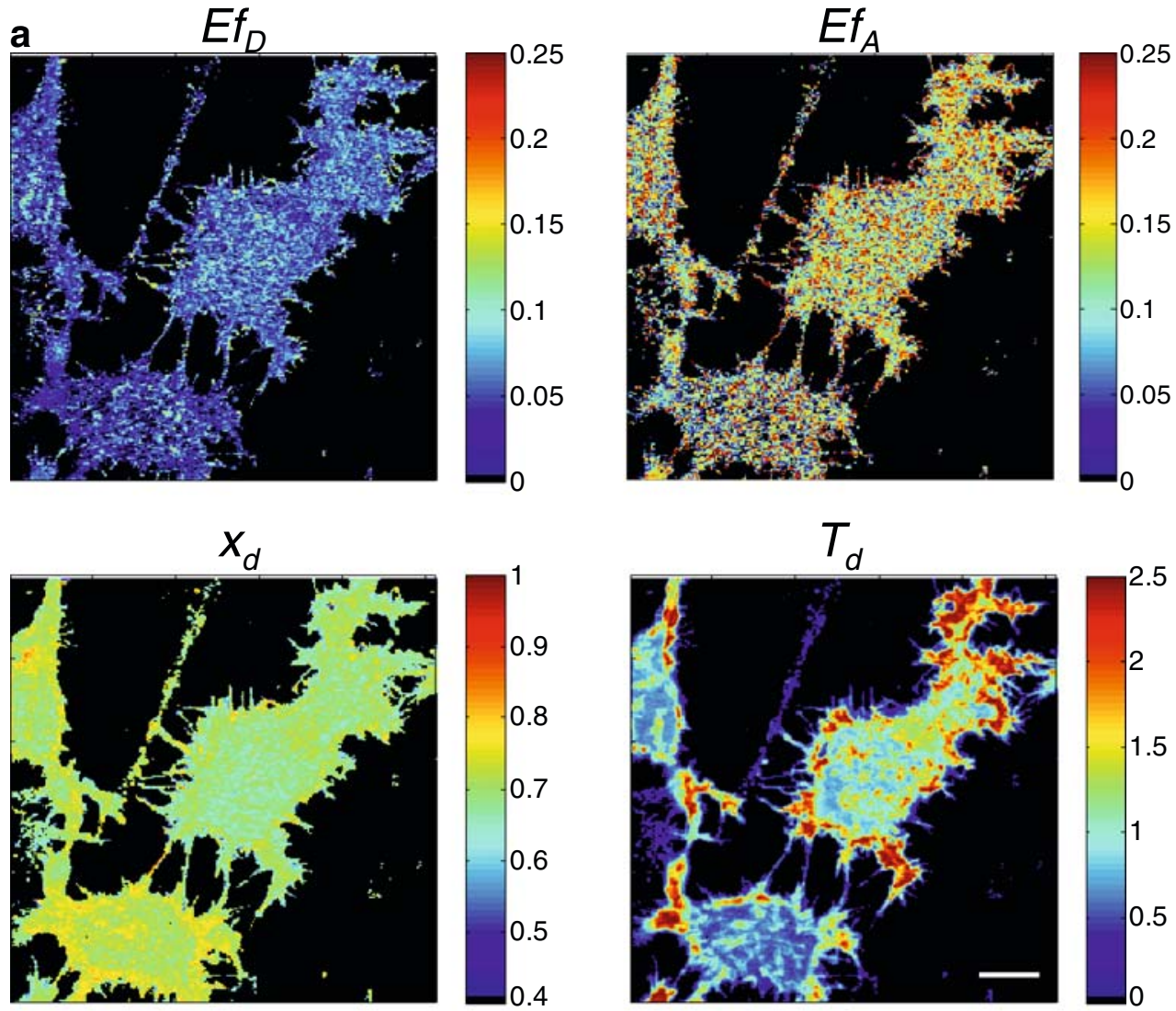

b

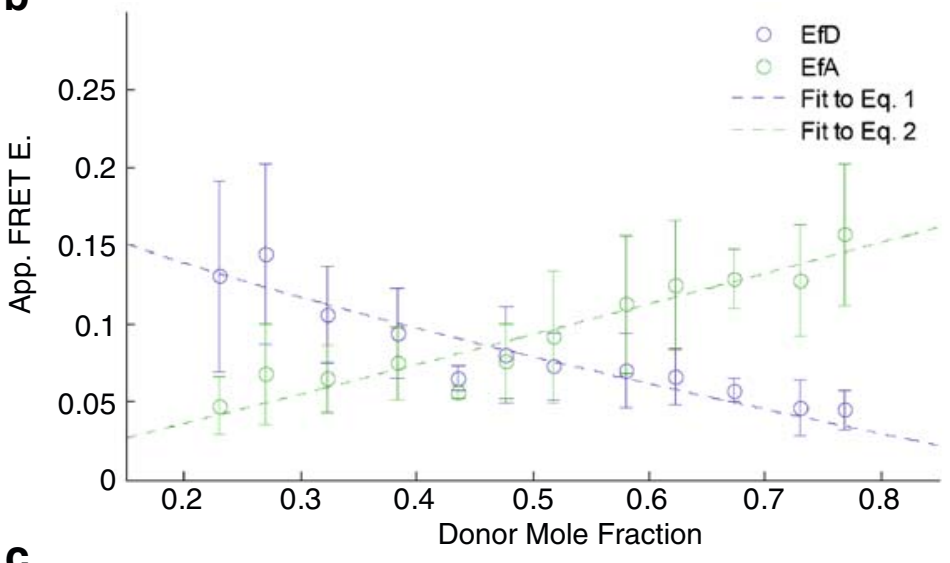

C

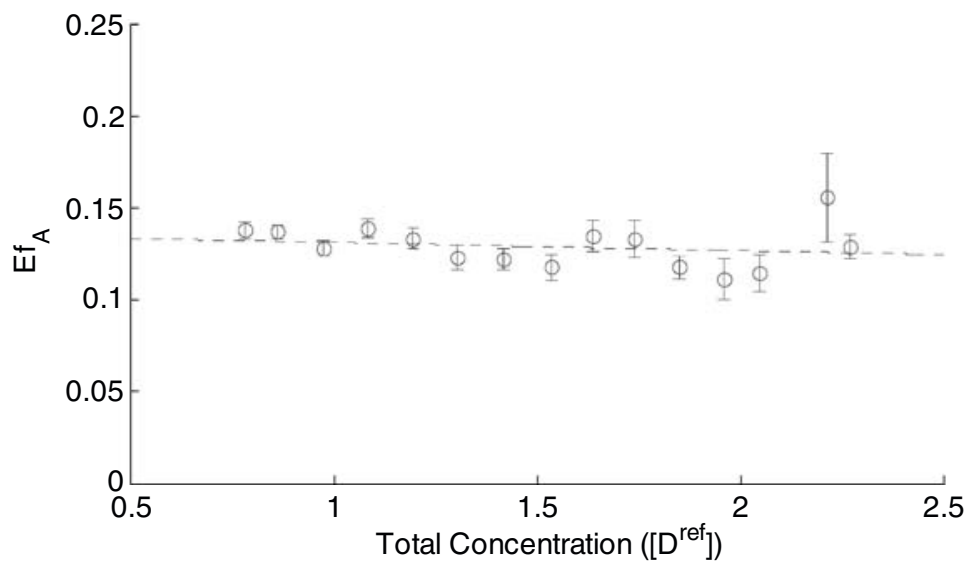


cells were measured for both wild-type and mutant receptors. Results obtained with a donor mole fraction below 0.2 and above 0.8 were excluded from the analysis due to error resulting from excessive differential bleaching between CFP and YFP.

Figure 2A shows the spatial distribution of the apparent FRET efficiencies $\left(E f_{D}\right.$ and $\left.E f_{A}\right)$, the donor mole fraction, as well as the relative total concentration at the plasma membrane of the cell co-transfected with the CFP-and YFP-tagged wild-type receptors. Based on these results we were able to build the relationship between the apparent FRET efficiencies and the donor mole fraction, $x_{D}$. Analysis of the data according to the aforementioned model reveals that the best fit for the $E f_{D}$ data was achieved with values of $E=0.11$ and $n=2.95\left(R^{2}=0.69\right)$. The best fit for the $E f_{A}$ data was achieved with $E=0.16$ and $n=2.44\left(R^{2}=0.83\right)$. Taken together these data suggest that in the case of the wild-type 5-HT1A receptor, more than two units participate in the formation of oligomers. However, due to the limitations of the model, we cannot exactly define the n-value.

To analyze the role of receptor palmitoylation for the oligomerization at the plasma membrane, we performed similar single-cell FRET analysis for the cells expressing CFP-and YFP-tagged acylation-deficient mutants (Fig. 3A). Comparison of the panel A in Figs. 2 and 3 does not reveal any significant differences in receptor or FRET distribution at the plasma membrane between wild-type and mutated receptors. These figures also demonstrate that the overall levels of the FRET for the cases of wild-type and nonpalmitoylated receptor expression were comparable. However, we obtained a slight difference in the relationship of FRET to the donor mole fraction. In the case of the mutant receptor, the limiting value of $E f_{D}$ approaches the same value as $E f_{A}$, while with the wild-type receptor the limit of the $E f_{A}$ value was greater than that of the $E f_{D}$. Fitting the data according to the oligomerization model for the $E f_{D}$ produced the value of $E=0.21$ and $n=1.70\left(R^{2}=0.8641\right)$ and for $E f_{A}$-values of $E=0.17$ and $n=2.10\left(R^{2}=0.8874\right)$ for the non-palmitoylated receptors.

It is also notable that the goodness of fit of the model to the wild-type receptor data is lower than to the mutant receptor data. The previously mentioned deficiency in the model may result in a less than ideal fit for data measured from a system with complexes composed of more than two units, as is the suggested case for the wild-type receptor.

Differences between the E values obtained for wild-type and mutated receptors suggest that non-palmitoylated receptor possesses a higher characteristic efficiency of energy transfer or a higher percentage of receptors are involved in oligomerization. Palmitoylation of the 5-HT1A receptor has been suggested to be responsible for the creation of an additional fourth intracellular loop at the receptor C-terminus [5]. The non-palmitoylated mutant will lack this loop and, therefore, may adopt a conformation which is more favorable for the FRET between the $\mathrm{C}$ terminally fused fluorophores.

In the case of the acylation-deficient mutant, the oligomeric complexes were suggested to be formed by two interacting units ( $n=1.7$ and $n=2.10$, for the $E f_{D}$ and $E f_{A}$ fits, respectively), while in the wild-type receptor more than two units can be involved in complex formation $(n=$ 2.95 and $n=2.44$, for the $E f_{D}$ and $E f_{A}$ fits, respectively). One possible explanation for such differences is the differential distribution of wild-type and mutant receptors within the plasma membrane. We have previously demonstrated that significant fraction of the 5-HT1A receptor resides in lipid rafts, while the yield of the palmitoylationdeficient receptor in these membrane microdomains is considerably reduced [24]. Furthermore, it has been shown that localization of proteins in lipid rafts may significantly increase stochastic interaction [28]. Therefore, the suggested greater number of units participating in complex obtained from the model for the wild-type receptors may results from stochastic interaction between oligomeric complexes with free receptors and/or with another complex localized in lipid microdomain. In contrast, nonpalmitoylated receptors, which are excluded from lipid rafts [24], have a much lower probability for the additional interaction.

The next important question addressed in the present study was the analysis of interaction specificity. It has been reported that positive FRET signals may result not only from specific protein interactions, including receptor oligomerization, but also from randomly distributed and overexpressed FRET donors and acceptors. Based on the critical analysis of BRET data, it has been recently considered that the contribution of non-specific interactions measured by resonance energy transfer may be even larger than previously thought [32]. Interaction specificity may be analyzed by plotting energy transfer efficiency as a function of expression level at fixed donor/acceptor ratio [32]. In the case of random interaction it has been proposed that the energy transfer between two fluorophores is linearly dependent on expression level and will diminish to zero at very low concentration of fluorophores. In the case of nonrandom interaction, the apparent FRET efficiency should be relatively independent of concentration with an $E f_{A}$ intercept greater than zero. Figures $2 \mathrm{C}$ and $3 \mathrm{C}$ show the relationship between apparent FRET efficiency $E f_{A}$ and total surface concentration $T_{D}$ obtained for the wild-type and acylation-deficient receptors at fixed donor to acceptor ratio. Both Figs. $2 \mathrm{C}$ and $3 \mathrm{C}$ demonstrate that FRET obtained for 5-HT1A receptors was largely independent of expression level, confirming non-random interactions between the receptors. 
Acknowledgements These studies were supported by the fund of the Medical School at the University of Göttingen and by the Deutsche Forschungsgemeinschaft through the Center of Molecular Physiology of the Brain to E.G.P., Grant PO 732 and a Georg Christoph Lichtenberg Stipend to A.W.

\section{References}

1. Wess, J.: G-protein-coupled receptors: molecular mechanisms involved in receptor activation and selectivity of G-protein recognition. FASEB J. 11, 346-354 (1997)

2. Wheatley, M., Hawtin, S.R.: Glycosylation of G-protein-coupled receptors for hormones central to normal reproductive functioning: its occurrence and role. Hum. Reprod. Update 5, 356-364 (1999). doi:10.1093/humupd/5.4.356

3. Qanbar, R., Bouvier, M.: Role of palmitoylation/depalmitoylation reactions in G-protein-coupled receptor function. Pharmacol. Ther. 97, 1-33 (2003). doi:10.1016/S0163-7258(02)00300-5

4. Resh, M.D.: Palmitoylation of ligands, receptors, and intracellular signaling molecules. Sci. STKE 2006(359), re14 (2006)

5. Papoucheva, E., Dumuis, A., Sebben, M., Richter, D.W., Ponimaskin, E.G.: The 5-hydroxytryptamine(1A) receptor is stably palmitoylated, and acylation is critical for communication of receptor with Gi protein. J. Biol. Chem. 279, 3280-3291 (2004). doi:10.1074/jbc.M308177200

6. Devi, L.A.: Heterodimerization of G-protein-coupled receptors: pharmacology, signaling and trafficking. Trends Pharmacol. Sci. 22, 532-537 (2001). doi:10.1016/S0165-6147(00)01799-5

7. Javitch, J.A.: The ants go marching two by two: oligomeric structure of G-protein-coupled receptors. Mol. Pharmacol. 66, 1077-1082 (2004). doi:10.1124/mol.104.006320

8. Lopez-Gimenez, J.F., Canals, M., Pediani, J.D., Milligan, G.: The alpha1b-adrenoceptor exists as a higher-order oligomer: effective oligomerization is required for receptor maturation, surface delivery, and function. Mol. Pharmacol. 71, 1015-1029 (2007). doi:10.1124/mol.106.033035

9. van Rijn, R.M., Chazot, P.L., Shenton, F.C., Sansuk, K., Bakker, R.A., Leurs, R.: Oligomerization of recombinant and endogenously expressed human histamine H(4) receptors. Mol. Pharmacol. 70, 604-615 (2006). doi:10.1124/mol.105.020818

10. Angers, S., Salahpour, A., Bouvier, M.: Biochemical and biophysical demonstration of GPCR oligomerization in mammalian cells. Life Sci. 68, 2243-2250 (2001). doi:10.1016/S00243205(01)01012-8

11. Pfleger, K.D., Eidne, K.A.: Monitoring the formation of dynamic G-protein-coupled receptor-protein complexes in living cells. Biochem. J. 385, 625-637 (2005). doi:10.1042/BJ20041361

12. Bulenger, S., Marullo, S., Bouvier, M.: Emerging role of homoand heterodimerization in G-protein-coupled receptor biosynthesis and maturation. Trends Pharmacol. Sci. 26, 131-137 (2005). doi:10.1016/j.tips.2005.01.004

13. Jones, K.A., Borowsky, B., Tamm, J.A., Craig, D.A., Durkin, M. M., Dai, M., et al.: GABA(B) receptors function as a heteromeric assembly of the subunits GABA(B)R1 and GABA(B)R2. Nature 396, 674-679 (1998). doi:10.1038/25348

14. Kaupmann, K., Schuler, V., Mosbacher, J., Bischoff, S., Bittiger, H., Heid, J., et al.: Human gamma-aminobutyric acid type B receptors are differentially expressed and regulate inwardly rectifying $\mathrm{K}+$ channels. Proc. Natl. Acad. Sci. U S A 95, 14991-14996 (1998). doi:10.1073/pnas.95.25.14991

15. Maggio, R., Novi, F., Scarselli, M., Corsini, G.U.: The impact of G-protein-coupled receptor hetero-oligomerization on function and pharmacology. FEBS J. 272, 2939-2946 (2005). doi:10.1111/j.1742-4658.2005.04729.x
16. Milligan, G.: G-protein-coupled receptor heterodimers: pharmacology, function and relevance to drug discovery. Drug Discov. Today 11, 541-549 (2006). doi:10.1016/j.drudis.2006.04.007

17. White, J.H., Wise, A., Main, M.J., Green, A., Fraser, N.J., Disney, G.H., et al:: Heterodimerization is required for the formation of a functional GABA(B) receptor. Nature 396, 679-682 (1998). doi: $10.1038 / 25354$

18. Foster, L.J., De Hoog, C.L., Mann, M.: Unbiased quantitative proteomics of lipid rafts reveals high specificity for signaling factors. Proc. Natl. Acad. Sci. U S A 100, 5813-5818 (2003). doi:10.1073/pnas.0631608100

19. Okamoto, T., Schlegel, A., Scherer, P.E., Lisanti, M.P.: Caveolins, a family of scaffolding proteins for organizing "preassembled signaling complexes" at the plasma membrane. J. Biol. Chem. 273, 5419-5422 (1998). doi:10.1074/jbc.273.10.5419

20. Simons, K., Toomre, D.: Lipid rafts and signal transduction. Nat. Rev. Mol. Cell Biol. 1, 31-39 (2000). doi:10.1038/35036052

21. Moffett, S., Brown, D.A., Linder, M.E.: Lipid-dependent targeting of G proteins into rafts. J. Biol. Chem. 275, 2191-2198 (2000). doi:10.1074/jbc.275.3.2191

22. Pucadyil, T.J., Kalipatnapu, S., Chattopadhyay, A.: Membrane organization and dynamics of the G-protein-coupled serotonin1A receptor monitored using fluorescence-based approaches. J. Fluoresc. 15, 785-796 (2005). doi:10.1007/s10895-005-2988-2

23. Zacharias, D.A., Violin, J.D., Newton, A.C., Tsien, R.Y.: Partitioning of lipid-modified monomeric GFPs into membrane microdomains of live cells. Science 296, 913-916 (2002). doi:10.1126/science.1068539

24. Renner, U., Glebov, K., Lang, T., Papusheva, E., Balakrishnan, S., Keller, B., et al.: Localization of the mouse 5-hydroxytryptamine (1A) receptor in lipid microdomains depends on its palmitoylation and is involved in receptor-mediated signaling. Mol. Pharmacol. 72, 502-513 (2007). doi:10.1124/mol.107. 037085

25. Kobe, F., Renner, U., Woehler, A., Wlodarczyk, J., Papusheva, E., Bao, G., et al:: Stimulation-and palmitoylation-dependent changes in oligomeric conformation of serotonin 5-HT1A receptors. Biochim. Biophys. Acta. 1783, 1503-1516 (2008)

26. Wlodarczyk, J., Woehler, A., Kobe, F., Ponimaskin, E., Zeug, A., Neher, E.: Analysis of FRET signals in the presence of free donors and acceptors. Biophys. J. 94, 986-1000 (2008). doi:10.1529/ biophysj.107.111773

27. Veatch, W., Stryer, L.: The dimeric nature of the gramicidin A transmembrane channel: conductance and fluorescence energy transfer studies of hybrid channels. J. Mol. Biol. 113, 89-102 (1977). doi:10.1016/0022-2836(77)90042-0

28. Meyer, B.H., Segura, J.M., Martinez, K.L., Hovius, R., George, N., Johnsson, K., et al.: FRET imaging reveals that functional neurokinin- 1 receptors are monomeric and reside in membrane microdomains of live cells. Proc. Natl. Acad. Sci. U S A 103, 2138-2143 (2006). doi:10.1073/pnas.0507686103

29. Harrison, C., van der Graaf, P.H.: Current methods used to investigate $\mathrm{G}$ protein coupled receptor oligomerisation. J. Pharmacol. Toxicol. Methods 54, 26-35 (2006). doi:10.1016/j. vascn.2005.11.001

30. Hoyer, D., Pazos, A., Probst, A., Palacios, J.M.: Serotonin receptors in the human brain. I. Characterization and autoradiographic localization of 5-HT1A recognition sites. Apparent absence of 5-HT1B recognition sites. Brain Res. 376, 85-96 (1986). doi:10.1016/0006-8993(86)90902-9

31. Pazos, A., Palacios, J.M.: Quantitative autoradiographic mapping of serotonin receptors in the rat brain. I. Serotonin-1 receptors. Brain Res. 346, 205-230 (1985). doi:10.1016/0006-8993(85)90856-X

32. James, J.R., Oliveira, M.I., Carmo, A.M., Iaboni, A., Davis, S.J.: A rigorous experimental framework for detecting protein oligomerization using bioluminescence resonance energy transfer. Nat. Methods 3, 1001-1006 (2006). doi:10.1038/nmeth978 